\title{
Expression of Mcl-1 and p53 proteins predicts the survival of patients with T3 gastric carcinoma
}

\author{
Yoshiniko Maeta, Shunichi Tsujitani, Sachiko Matsumoto, Kenichi Yamaguchi, Shigeru Tatebe, \\ Akira Kondo, Masahide Ikeguchi, and Nobuaki Kaibara \\ Department of Surgery, Faculty of Medicine, Tottori University, 36-1 Nishi-cho, Yonago 683-8504, Japan
}

\begin{abstract}
Background. The expression of myeloid cell leukemia 1 (Mcl1) and p53 proteins was investigated for clinicopathological and prognostic significance in patients with gastric carcinoma. Methods. Mcl-1 protein was immunohistochemically examined in 182 patients with gastric carcinoma. The overexpression of p53 was also analyzed in T3 gastric carcinomas.

Results. The expression of Mcl-1 was detected in $127(69.8 \%)$ patients with gastric carcinoma. Mcl-1 was detected significantly more frequently in the undifferentiated type $(P<0.05)$ and in the advanced stage of disease $(P<0.05)$. The prognosis of patients with an Mcl-1-positive tumor was significantly worse than that of those with an Mcl-1-negative tumor $(P<0.05)$. Multivariate analysis revealed that the expression of Mcl-1 was an independent prognostic factor, as were lymph node metastasis and tumor size. There was no significant relationship between the expression of Mcl-1 and p53. In patients with T3 gastric carcinoma who underwent curative surgery; however, Mcl-1(-)/p53 (-) tumor demonstrated the best postoperative survival rate, whereas $\mathrm{Mcl}-1(+) / \mathrm{p53}(+)$ tumor had the worst.

Conclusion. The expression of Mcl-1 is an indicator of tumor progression and postoperative recurrence in patients with gastric carcinoma. Combined analysis of Mcl-1 and p53 proteins may accurately predict the survival of patients with $\mathrm{T} 3$ gastric carcinoma.
\end{abstract}

Key words Gastric cancer · Mcl-1 · p53 - Immunohistochemistry $\cdot$ Survival

\section{Introduction}

Apoptosis, or programmed cell death, is the main physiological mechanism of cell death, and is a highly con-

Offprint requests to: $\mathrm{Y}$. Maeta

Received: October 20, 2003 / Accepted: January 27, 2004 served, precisely regulated, mechanistically driven process, clearly distinct from necrosis [1]. If control of an active pathway that leads to apoptotic cell death is lost, the resulting imbalance between proliferation and apoptosis could enhance the potential for neoplastic growth. Alteration of a variety of oncogenes and tumor suppressor genes may ultimately result in an imbalance between proliferation and apoptosis, and lead to net tumor growth and progression [2,3].

In recent years, many of the genes involved in apoptosis have been identified and cloned $[4,5]$. The myeloid cell leukemia 1 (Mcl-1) gene is a member of the $B c l-2$ family and encodes for proteins that promote cell survival [6-8]. Some members of this family are blockers of cell death, including $B c l-2, B c l-X L$, and Mcl-1, whereas others, such as $B a x$ and $B c l-X s$, are promoters of apoptosis. Members of this family regulate apoptosis by forming either homodimers or heterodimers with each other, and the relative ratio of anti- to pro-apoptotic proteins determines cell sensitivity to apoptotic stimuli [9]. In light of its anti-apoptotic function, high expression levels of Mcl-1 in cancer cells may prolong cell survival, allow development and progression of tumor cells, and ultimately result in a poor prognosis.

In fact, Mcl-1 expression has been reported to be a poor prognostic marker in certain tumors. Shigemasa et al. [10] reported that expression of Mcl-1 significantly correlated with an advanced clinical stage, high histologic grade, and poor survival in ovarian cancer. In addition, recent studies have shown that the anti-apoptitic Bcl-2 family of proteins are involved in the mechanisms of tumor resistance to chemotherapeutic agents and radiation [11-13]. However, Mcl-1 expression in gastric carcinomas and its relationship to a patient's prognosis and other clinicopathological variables have yet to be clarified.

In the present study, the expression of Mcl-1 in surgically resected gastric carcinomas was examined, and 
its association with clinicopahological variables and a patient's prognosis was investigated.

\section{Patients, materials, and methods}

\section{Patients}

Primary gastric adenocarcinoma specimens were obtained from 182 patients treated surgically at our institution from 1982 to 1992 . The patients' ages ranged from 32 to 91 years (average, 62.4 years); 95 were male and 87 were female. None of the patients had received preoperative adjuvant radiotherapy or chemotherapy. The clinicopathological findings were determined according to the Japanese classification of gastric carcinoma [14]. The patients were classified as follows: 33 patients were in stage I, 46 in stage II, 80 in stage III, and 23 in stage IV. All had undergone either a distal partial gastrectomy, a proximal gastrectomy, or a total gastrectomy with regional lymph node dissection to group $1\left(\mathrm{D}_{1}\right)$, group $2\left(\mathrm{D}_{2}\right)$, and group $3\left(\mathrm{D}_{3}\right)$, excluding the paraaortic nodes, with curative intent. Most of the patients with an advanced tumor received postoperative chemotherapy with consecutive oral administration of uracil/tegafur, at a dose of $300 \mathrm{mg}$ daily, for 1 year after surgery. However, some patients refused or discontinued chemotherapy. The patients were periodically checked for early recurrence by diagnostic imaging (chest X-ray, double-contrast barium meal study, upper gastrointestinal fiberscopy, ultrasonography, and computed tomography). All of the patients were monitored for at least 5 years. Informed consent had been obtained from all subjects and/or their guardians.

\section{Immunohistochemistry}

Mcl-1 expression. For immunohistochemical analysis, a streptavidin-biotin method (Histofine SAB-PO kit; Nichirei, Tokyo, Japan) was used for formalin-fixed and paraffin-embedded specimens. Sections $(4 \mu \mathrm{m})$ were dewaxed in xylene, rehydrated in ethanol, and then heated in a microwave oven $(700 \mathrm{~W})$ for $20 \mathrm{~min}$ for retrieval of antigens. Endogenous peroxidase was blocked with $0.3 \%$ hydrogen peroxide in methanol for $15 \mathrm{~min}$. Tissue sections were then washed twice with phosphate-buffered saline (PBS) and preblocked for 60 min with $10 \%$ goat serum. After the washing with PBS, the samples were incubated overnight at $4^{\circ} \mathrm{C}$ with anti-Mcl-1 polyclonal antibody (sc-819; Santa Cruz Biotechnology, Santa Cruz, CA, USA). Sections were then washed three times in PBS and incubated with secondary anti-rabbit immunoglobulin (IgG 1 IgA 1 IgM) conjugated with biotin (Nichirei) for $60 \mathrm{~min}$, followed by incubation with a streptavidin-peroxidase complex (Nichirei) for another $60 \mathrm{~min}$. After three additional washes in PBS, diaminobenzidine tetrahydrochloride working solution was applied. Finally, the slides were counterstained in hematoxylin. The staining pattern was classified as follows: (1) staining was considered absent when there was no positive tumor cell staining or fewer than $5 \%$ focally distributed positive tumor cells; (2) labeling was considered weak when $5 \%$ to $50 \%$ of the tumor cells showed positive staining; and (3) labeling was considered strong when more than $50 \%$ of the tumor cells showed positive staining.

p53 Expression. Staining for p53 was performed using an anti-p53 monoclonal antibody (BP-53; Novocastra Laboratories, Newcastle, UK) at a dilution of $1: 50$. The methods used for immunostaining were as described previously [15]. The staining pattern was classified as follows: (2), negative or equivocal staining; (1), weak (positive expression was detected in 1\%-20\% of carcinoma cells); and (11), strong expression (positive expression was detected in .20\% of carcinoma cells). This classification was based on a previous report [16].

\section{Statistical analysis}

Statistical analysis included the standard $\chi^{2}$-test and the Mann-Whitney $U$-test. The survival rates were estimated by the Kaplan-Meier method, and statistical analysis was carried out by the log-rank test for equality of the survival curves. In multivariate analysis, independent prognostic factors were determined with the Cox proportional hazard model (StatView 5.0; Abacus Concepts, Berkeley, CA, USA). Statistical significance was considered with $P<0.05$.

\section{Results}

Mcl-1 was immunolocalized to the cytoplasm of cancer cells, but not in the underlying stromal cells (Fig. 1). However, weak and diffuse staining for Mcl-1 was also detected in the normal mucosal cells. Mcl-1 expression in cancer cells was assessed by comparison with expression levels in the normal mucosal cells used as the negative control. Slight immunoreactivity to anti-Mcl-1 antibody was judged as negative staining (Fig. 1B).

Mcl-1 expression was absent in 55 cases (30.2\%), weak in 46 cases $(25.3 \%)$, and strong in 81 cases $(44.5 \%)$. To analyze the correlation between Mcl-1 expression and clinicopathological features and prognosis, we considered that cases with fewer than 5\% Mcl-1-positive cancer cells (staining absent) were negative, and those with over $5 \%$ of Mcl-1-positive cancer cells (weak and strong labeling) were positive. Out of all 182 patients, Mcl-1 expres- 

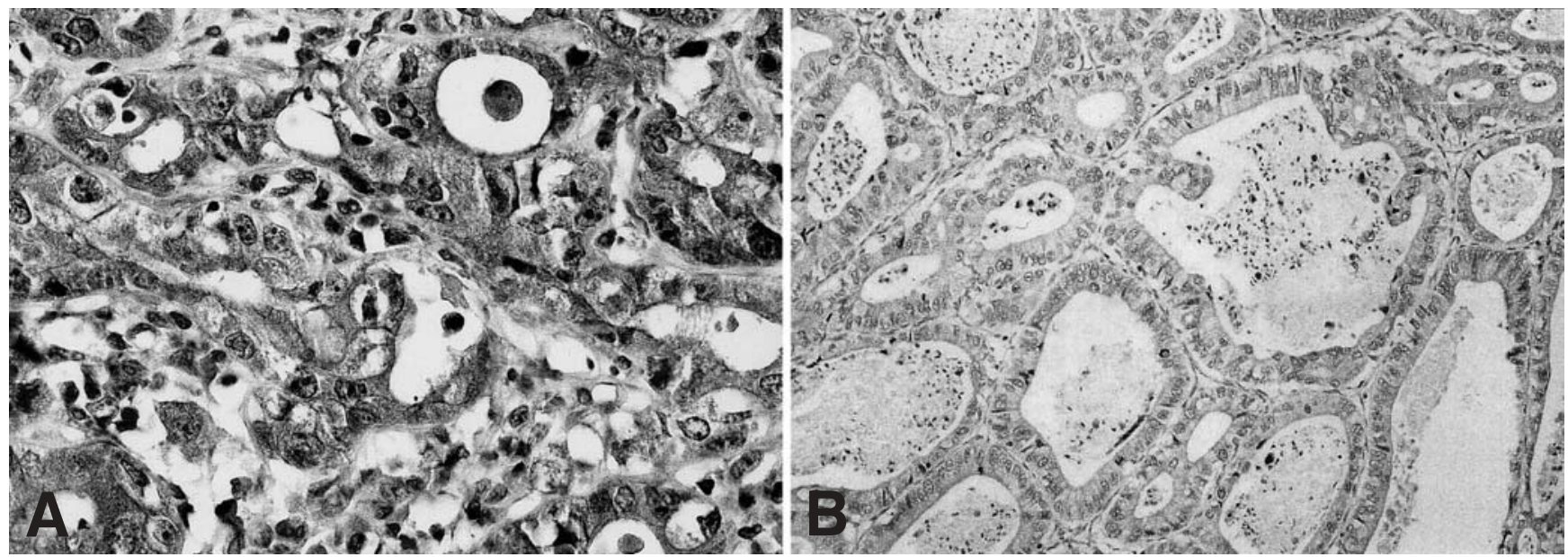

Fig. 1A,B. Typical results of myeloid cell leukemia 1 (Mcl-1) immunohistochemistry. A Mcl-1-positive case: cytoplasmic expression of Mcl-1 protein was detected in more than 5\% of tumor cells. B Mcl-1-negative case: no expression of Mcl-1 was found in tumor tissues $\mathbf{A} \times 400 ; \mathbf{B} \times 200$

sion was negative in $55(30.2 \%)$, and positive in 127 $(69.8 \%)$.

The correlation between Mcl-1 immunohistochemical expression and clinicopathological variables in the 182 patients is shown in Table 1 . Mcl-1 expression showed no correlation with age, sex, tumor size, lymph node metastasis, or lymphatic-vascular space invasion, whereas the expression was significantly more frequently detected in the undifferentiated type than the differentiated type $(P<0.05)$. Mcl-1 expression was significantly associated with advanced histological stage $(P<0.05$; Table 1).

The survival time was determined in 179 patients; 3 patients were excluded because they died of causes other than gastric cancer within 1 month of surgery. The estimated 5-year survival rates of patients with negative and positive Mcl-1 expression were $73.7 \%$ and $52.5 \%$, respectively. The prognosis for Mcl-1-positive patients was significantly poorer than that for the Mcl-1-negative patients (Fig. 2). The survival curves of patients, stratified by Mcl-1 expression for each histological stage, are shown in Fig. 3. In stage I, II, and IV patients, there was no significant correlation between the patient's prognosis and Mcl-1 expression. In stage III, however, patients with Mcl-1-positive tumors had a significantly lower survival rate than those with Mcl-1negative tumors $(P<0.05)$. Table 2 shows the results of the multivariate analysis with the Cox proportional hazards model in the 179 patients. The covariates included in the model were the patient's age, sex, tumor size, histological type, depth of invasion, lymph node metastasis, lymphatic invasion, venous invasion, and Mcl-1 expression. The analysis revealed that lymph node metastasis, tumor size, and Mcl-1 expression contributed independently to survival.
The survival rate of patients with gastric carcinoma invading the serosa (T3 gastric carcinoma) who underwent curative surgery was further analyzed. Patients with Mcl-1-positive tumors with lymph node metastasis had a significantly shorter survival time than those with Mcl-1-negative tumors $(P<0.05$; Fig. 4). However, Mcl-1 expression did not affect the survival of patients without lymph node metastasis.

In T3 gastric carcinoma, p53 expression was positive in 80 patients $(64 \%)$. There was no significant correlation between Mcl-1 and p53 expression. The 5-year survival rate in patients with a p53-negative tumor (77.2\%) was significantly better than that of those with a p53positive tumor $(48.1 \% ; P<0.01)$. The 125 patients with T3 gastric carcinoma who underwent curative surgery were divided into subgroups according to the Mcl-1 and p53 status of tumors as follows: $\mathrm{Mcl}-1(-) / \mathrm{p} 53(-), n=$ 16; $\operatorname{Mcl}-1(+) / \mathrm{p} 53(-), n=29 ; \operatorname{Mcl}-1(-) / \mathrm{p} 53(+), n=17$; and Mcl-1(+)/p53(+), $n=63$. The 5-year survival rates for these four groups were $93.3 \%, 68.6 \%, 58.8 \%$, and $45.2 \%$, respectively. Patients with Mcl-1(-)/p53(-) tumors demonstrated the best survival rate, whereas those with an Mcl-1(+)/p53(+) tumor had the worst (Fig. 5).

\section{Discussion}

In this study, we have demonstrated the frequent occurrence of Mcl-1 expression in surgically resected gastric carcinoma (127/182 cases were positive; $69.8 \%)$. As described above, Mcl-1 is a blocker of cell death. In light of its anti-apoptotic function, we hypothesized that patients with Mcl-1-positive gastric carcinoma had a poorer prognosis than those with Mcl-1-negative tumors. We also revealed a striking association between 
Table 1. Correlation between myeloid cell leukemia 1 (Mcl-1) expression and clinicopathological features

\begin{tabular}{|c|c|c|c|}
\hline Variables & Mcl-1-negative $(n=55)$ & Mcl-1-positive $(n=127)$ & $P$ value \\
\hline Age (years) & $61 \pm 12$ & $63 \pm 13$ & NS \\
\hline \multicolumn{4}{|l|}{ Sex } \\
\hline Male & $33(34.7 \%)$ & $62(65.3 \%)$ & \multirow[t]{2}{*}{ NS } \\
\hline Female & $22(25.3 \%)$ & $65(74.7 \%)$ & \\
\hline Tumor size $(\mathrm{cm})$ & $6.6 \pm 3.4$ & $7.9 \pm 4.6$ & NS \\
\hline \multicolumn{4}{|l|}{ Histology } \\
\hline Differentiated & $27(39.7 \%)$ & $41(60.3 \%)$ & \multirow[t]{2}{*}{$P<0.05$} \\
\hline Undifferentiated & $28(24.6 \%)$ & $86(75.4 \%)$ & \\
\hline \multicolumn{4}{|c|}{ Lymph node metastasis } \\
\hline Absent & $24(30.0 \%)$ & $56(70.0 \%)$ & \multirow[t]{2}{*}{ NS } \\
\hline Present & $31(30.4 \%)$ & $71(69.6 \%)$ & \\
\hline \multicolumn{4}{|l|}{ Lymphatic invasion } \\
\hline Absent & $27(36.0 \%)$ & $48(64.0 \%)$ & \multirow[t]{2}{*}{ NS } \\
\hline Present & $28(26.2 \%)$ & $79(73.8 \%)$ & \\
\hline \multicolumn{4}{|l|}{ Vascular invasion } \\
\hline Absent & $25(34.2 \%)$ & $48(65.8 \%)$ & \multirow[t]{2}{*}{ NS } \\
\hline Present & $30(27.5 \%)$ & $79(72.5 \%)$ & \\
\hline \multicolumn{4}{|l|}{ Histological stage } \\
\hline I & $16(48.5 \%)$ & $17(51.5 \%)$ & \multirow[t]{4}{*}{$P<0.05$} \\
\hline II & $8(17.4 \%)$ & $38(82.6 \%)$ & \\
\hline III & $25(31.3 \%)$ & $55(68.8 \%)$ & \\
\hline IV & $6(26.1 \%)$ & $17(73.9 \%)$ & \\
\hline
\end{tabular}

NS, not significant

Table 2. Multivariate analysis of prognostic factors by the Cox proportional hazards model

\begin{tabular}{lccr}
\hline Variables & Hazard ratio & $95 \%$ CI & $P$ value \\
\hline Lymph node metastasis & 4.385 & $2.107-9.124$ & $<0.0001$ \\
Tumor size & 1.928 & $1.142-3.256$ & 0.0141 \\
Mcl-1 expression & 2.015 & $1.094-3.710$ & 0.0245
\end{tabular}

CI, confidence interval

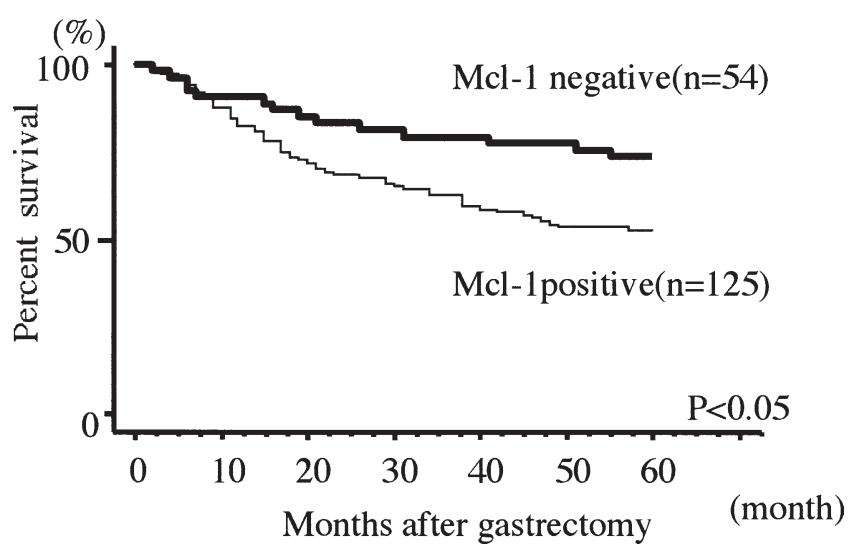

Fig. 2. Survival curve of 179 patients with gastric cancer according to Mcl-1 status. The prognosis of Mcl-1-positive patients was significantly worse than that of Mcl-1-negative patients $(P<0.05)$

increased Mcl-1 expression and a poor prognosis for patients with gastric carcinoma. Baekelandt et al. [17] have previously reported that immunohistochemical expression of Mcl-1 was significantly associated with a poor prognosis in stage III ovarian cancer. Also, Rieger et al. [18] reported that Mcl-1 expression was associated with the early recurrence of tumors and a shorter survival rate in patients with malignant glioma. To our knowledge, this is the first study to determine whether the patient's prognosis is significantly correlated with Mcl-1 expression in gastric carcinoma.

In this study, the percentage of Mcl-1-positive tumors in $\mathrm{T} 1$ tumors was $51.5 \%$, compared with $73.8 \%$ in $\mathrm{T} 2-3$ tumors. Mcl-1 expression was significantly correlated with histological stage. Therefore, it is possible that Mcl-1 expression could be related to cell proliferation and tumor progression. In support of our findings, Krajewska et al. [19-21] showed expression of the Bcl-2 family of proteins in the carcinogenesis of colorectal cancer and prostate cancer, and in the tumor progression of gastric cancer. In the present study, we further analyzed the relationship between patient survival rate and Mcl-1 expression to determine the factors involved in the recurrence of gastric cancer. When the survival rate was analyzed in each histological stage, there was a significant correlation between prognosis and Mcl-1 

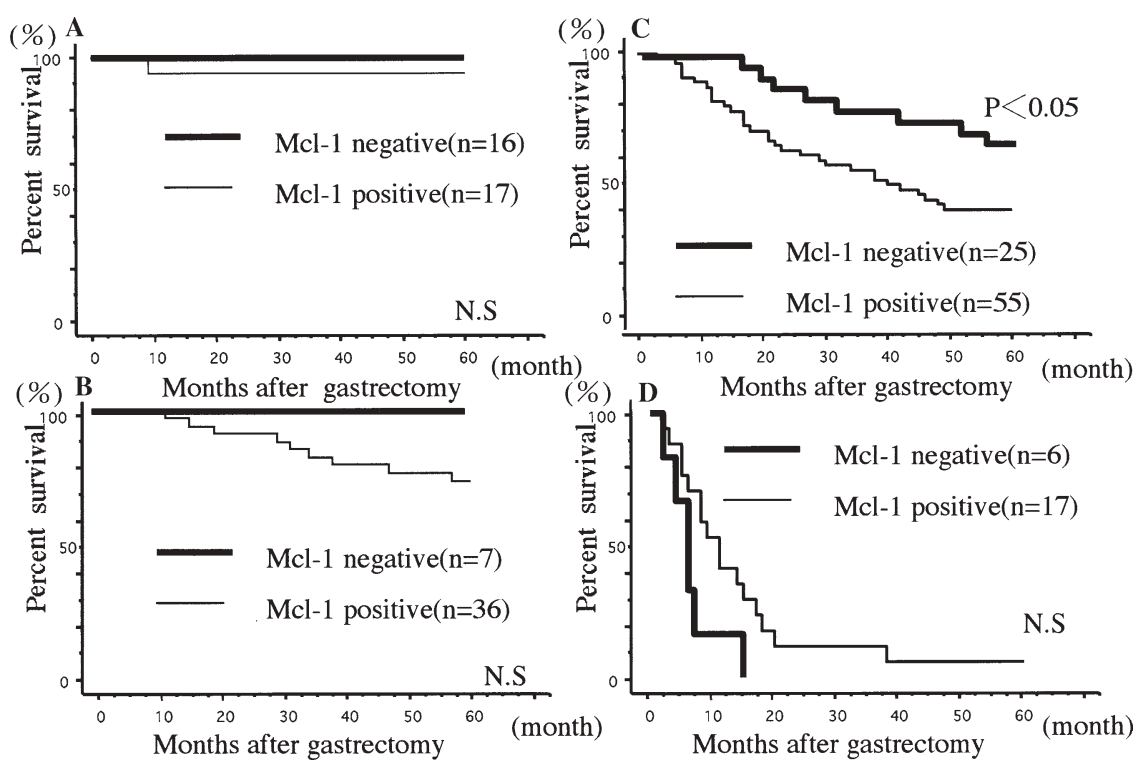

Fig. 3A-D. Survival curves, for each histological stage, in patients with gastric cancer: A stage I, B stage II, C stage III, D stage IV. There was no significant difference in stages I, II, and IV. In stage III, the prognosis of patients with an Mcl-1positive tumor was significantly poorer than that of those with an Mcl-1-negative tumor $(P<0.05)$. N.S., not significant

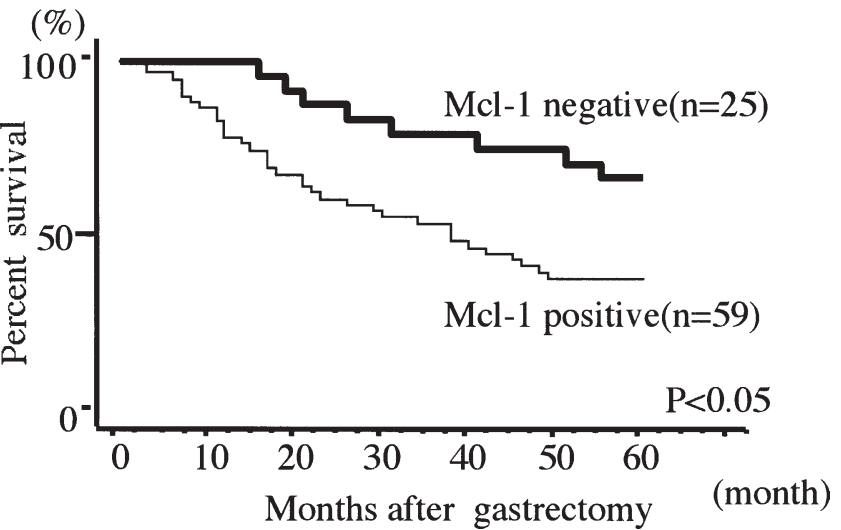

Fig. 4. Survival curves for patients with $\mathrm{T} 3$ gastric carcinoma with lymph node metastasis. The prognosis of Mcl-1-positive patients was significantly worse than that of Mcl-1-negative patients $(P<0.05)$

expression in stage III disease. Multivariate analysis revealed the significance of the immunohistochemical evaluation of Mcl-1 as an independent prognostic factor, along with lymph node metastasis and tumor size. These results indicate that Mcl-1 expression can be considered to be a tumor progression-related factor and a prognostic factor in gastric carcinoma.

Because of the relation of Mcl-1 to tumor progression, the number of Mcl-1-positive patients was obviously larger than that of Mcl-1-negative patients in the advanced stage. Serosal invasion is thought to be an important factor for peritoneal dissemination, the most frequent pattern of recurrence in advanced gastric carcinoma. Therefore, further analysis was done in patients with T3 gastric carcinoma. Patients with Mcl-1-positive T3 gastric carcinoma showed shorter survival than those with Mcl-1-negative tumors in those patients with

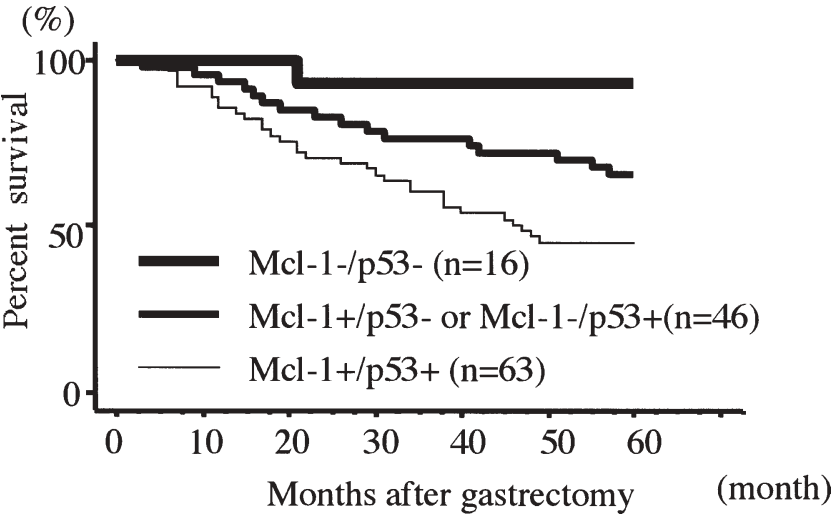

Fig. 5. Combined analysis, with Mcl-1 and p53 expression, of patients with T3 gastric carcinoma who underwent curative surgery. The survival curve of patients with Mcl-1+/p53+ tumors was significantly worse than those of the other groups

lymph node metastasis. Previously, we have reported that nuclear p53 accumulation correlates with tumor angiogenesis through vascular endothelial growth factor (VEGF) upregulation in gastric carcinoma [15]. In the present study, Mcl-1 expression was not correlated with p53 expression. Considering that Mcl-1 expression was not correlated with lymphatic-vascular space invasion or with lymph node metastasis, Mcl-1 expression may play an important role in the peritoneal recurrence of gastric carcinoma.

The Bcl-2 oncoprotein has been shown to prolong cell survival by inhibiting apoptosis [22]. A number of studies have reported that Bcl-2 expression in a variety of solid tumors is involved in carcinogenesis, tumor progression, and prognostic factors. Since the initial discovery of Bcl-2, several exclusively homologous proteins have been identified, revealing the presence of a 
multigene family. These Bcl-2 family members form homo- or heterodimers with each other, and these complex protein-protein associations program cells towards either survival or death, although the precise mechanisms of these processes remain unclear [4,5]. The $\mathrm{Mcl}$ 1 gene was first identified by the screening of cDNA libraries derived from a human myeloid leukemia cell line that was induced to undergo differentiation in culture. The amino-acid sequence of the human Mcl-1 protein reveals a polypeptide of 350 amino acids that is $35 \%$ homologous to Bcl-2. Recent studies have focused on the balance of expression of Bcl-2-related protein. Moreover, Mcl-1 can be upregulated by a variety of cytokines, such as interleukin (IL)-5, IL-6, IL-10, IL-13, and alpha-interferon [23-28]. A number of signaling pathways have been implicated in regulating the expression of Mcl-1 in a variety of experimental models $[24,25,29]$. Continuous activation of the pertinent signaling pathways is therefore likely to be important in maintaining the high levels of Mcl-1 expression. We analyzed the relationship between the expression of Mcl-1 and p53 in $\mathrm{T}_{3}$ gastric carcinoma. Khoury et al. [30] have reported that expression of Mcl-1 in mantle cell lymphoma is associated with overexpression of p53. In our present study, however, there was no statistically significant relationship between these factors, although overexpression of Mcl-1 and p53 proteins seemed to independently affect the prognosis. Combined analysis with Mcl-1 and p53 may provide an accurate prediction of the survival of patients with T3 gastric carcinoma.

In conclusion, we have found that a high expression level of Mcl-1 protein was significantly correlated with a poor survival rate in patients with gastric carcinoma, especially in the advanced stages. Furthermore, it is possible that Mcl-1 expression could be related to cell proliferation, and involved in recurrence, in patients with advanced gastric carcinoma. Combined analysis of Mcl-1 and p53 proteins may accurately predict the survival of patients with T3 gastric carcinoma. The fact that immunohistochemical analysis does not provide information on the functional status of the apoptosisregulatory protein, is a significant drawback. However, the preliminary findings of this report suggest that further study is required into the role of Mcl-1 in gastric cancer.

\section{References}

1. Kerr JF, Wyllie AH, Currie AR. Apoptosis:a basic biological phenomenon with wide-ranging implications in tissue kinetics. $\mathrm{Br}$ J Cancer 1972;26:239-57.

2. Manning FC, Patierno SR. Apoptosis:inhibitor or instigator of carcinogenesis? Cancer Invest 1996;14:455-65.

3. Korsmeyer SJ. Bcl-2 initiates a new category of oncogenes: regulators of cell death. Blood 1992;80:879-86.
4. Kroemer G. The proto-oncogene Bcl-2 and its role in regulating apoptosis. Nat Med 1997;3:614-20.

5. Korsmeyer SJ. BCL-2 gene family and the regulation of programmed cell death. Cancer Res 1999;59(Suppl):1693-700.

6. Reynolds JE, Li J, Craig RW, Eastman A. BCL-2 and MCL-1 expression in Chinese hamster ovary cells inhibits intracellular acidification and apoptosis induced by staurosporine. Exp Cell Res 1996;225:430-6.

7. Lomo J, Smeland EB, Krajewski S, Reed JC, Blomhoff HK. Expression of the $\mathrm{Bcl}-2$ homologue Mcl-1 correlates with survival of peripheral blood B lymphocytes. Cancer Res 1996;56:40-3.

8. Zhou P, Qian L, Kozopas KM, Craig RW. Mcl-1, a Bcl-2 family member, delays the death of hematopoietic cells under a variety of apoptosis-inducing conditions. Blood 1997;89:630-43.

9. Yang E, Korsmeyer SJ. Molecular thanatopsis: a discourse on the BCL2 family and cell death. Blood 1996;88:386-401.

10. Shigemasa K, Katoh O, Shiroyama Y, Mihara S, Mukai K, Nagai $\mathrm{N}$, et al. Increased MCL-1 expression is associated with poor prognosis in ovarian carcinomas. Jpn J Cancer Res 2002;93:54250.

11. Dole MG, Jasty R, Cooper MJ, Thompson CB, Nunez G, Castle VP. Bcl-xL is expressed in neuroblastoma cells and modulates chemotherapy-induced apoptosis. Cancer Res 1996;55:2576-82.

12. Gauthier ER, Piche L, Lemieux G, Lemieux R. Role of bcl-X(L) in the control of apoptosis in murine myeloma cells. Cancer Res 1996;56:1451-6.

13. Han Z, Chatterjee D, Early J, Pantazis P, Hendrickson EA, Wyche JH. Isolation and characterization of an apoptosisresistant variant of human leukemia HL-60 cells that has switched expression from Bcl-2 to Bcl-xL. Cancer Res 1996;56:1621-8.

14. Japanese Research Society for Gastric Cancer. Japanese classification of gastric carcinoma. First English Ed. Tokyo: Kanehara; 1995.

15. Saito H, Tujitani S, Ikeguchi M, Maeta M, Kaibara N. Neoangiogenesis and relationship to nuclear p53 accumulation and vascular endothelial growth factor expression in advanced gastric carcinoma. Oncology 1997;57:164-72.

16. Gomyo Y, Ikeda M, Ozaki M, Tatebe S, Tsujitani S, Ikeguchi M, et al. Expression of $\mathrm{p} 21$ (waf1/cip1/sdi1), but not p53 protein, is a factor in the survival of patients with advanced gastric carcinoma. Cancer 1997;79:2067-72.

17. Baekelandt M, Horm R, Nesland JM, Trope CG, Kristensen GB. Expression of apoptosis-related proteins is an independent determinant of patient prognosis in advanced ovarian cancer. J Clin Oncol 2000;18:3775-81.

18. Rieger L, Weller M, Bornemann A, Schabet M, Dichgans J, Meyermann R. BCL-2 family protein expression in human malignant glioma: a clinical-pathological correlative study. J Neurol Sci 1998;155:68- 75.

19. Krajewska M, Krajewski S, Epstein JI, Shabaik A, Sauvageot J, Song K, et al. Immunohistochemical analysis of bcl-2, bax, bcl-X, and mcl-1 expression in prostate cancer. Am J Pathol 1996; 148:1567-76.

20. Krajewska M, Moss SF, Krajewski S, Song K, Holt PR, Reed JC. Elevated expression of $\mathrm{Bcl}-\mathrm{X}$ and reduced Bak in primary colorectal adenocarcinomas. Cancer Res 1996;56:2422-7.

21. Krajewska M, Fenoglio-Preiser CM, Krajewski S, Song K, Macdonald JS, Stemmerman G, et al. Immunohistochemical analysis of Bcl-2 family proteins in adenocarcinomas of the stomach. Am J Pathol 1996;149:1449-57.

22. Korsmeyer SJ. Bcl-2 initiates a new category of oncogene. regulators of cell death. Blood 1992;80:879-86.

23. Puthier D, Bataille R, Amiot M. IL-6 up-regulates mcl- 1 in human myeloma cells through JAK/STAT rather than ras/MAP kinase pathway. Eur J Immunol 1999;29:3945-50.

24. Wei LH, Kuo ML, Chen CA, Chou CH, Cheng WF, Chang MC, et al. The anti-apoptotic role of interleukin-6 in human cervical cancer is mediated by up-regulation of Mcl-1 through a PI 3-K/ Akt pathway. Oncogene 2001;20:5799-809. 
25. Huang HM, Huang CJ, Yen JJ. Mcl-1 is a common target of stem cell factor and interleukin-5 for apoptosis prevention activity via MEK/MAPK and PI-3K/Akt pathways. Blood 2000; 96:1764-71.

26. Li L, Krajewski S, Reed JC, Choi YS. The apoptosis and proliferation of SAC-activated B cells by IL-10 are associated with changes in Bcl-2, Bcl-xL, and Mcl-1 expression. Cell Immunol 1997;178:33-41.

27. Lomo J, Blomhoff HK, Jacobsen SE, Krajewski S, Reed JC, Smeland EB. Interleukin-13 in combination with CD40 ligand potently inhibits apoptosis in human B lymphocytes: upregulation of Bcl-xL and Mcl-1. Blood 1997;89:4415-24.

28. Kuo ML, Chuang SE, Lin MT, Yang SY. The involvement of PI 3-K/Akt- dependent up-regulation of Mcl-1 in the prevention of apoptosis of Hep3B cells by interleukin-6. Oncogene 2001; 20:677-85.

29. Wang JM, Chao JR, Chen W, Kuo ML, Yen JJ, Yang-Yen HF, The antiapoptotic gene mcl-1 is up-regulated by the phosphatidylinositol 3-kinase/Akt signaling pathway through a transcription factor complex containing CREB. Mol Cell Biol 1999;19:6195206.

30. Khoury JD, Medeiros LJ, Rassidakis GZ, McDonnell TJ, Abruzzo LV, Lai R. Expression of Mcl-1 in mantle cell lymphoma is associated with high-grade morphology, a high proliferative state, and p53 overexpression. J Pathol 2003;199:90-7. 